\title{
Weissenbacher-Zweymuller syndrome
}

INSERM

\section{Source}

INSERM. (1999). Orphanet: an online rare disease and orphan drug data base.

Weissenbacher-Zweymuller syndrome. ORPHA:3450

Weissenbacher-Zweymuller syndrome (WZS) is characterized by short stature at birth, neonatal micrognathia, cleft palate, rhizomelic chondrodysplasia with 'dumbbell' shaped arm and leg bones, hypertelorism and vertebral coronal clefts. 